\section{ORIGINAL RESEARCH}

G. Hwang

E.-A. Jeong

J.H. Sohn

H. Park

J.S. Bang

S.-C. Jin

B.C. Kim

C.W. Oh

O.-K. Kwon

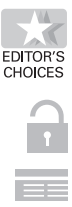

\title{
The Characteristics and Risk Factors of Headache Development after the Coil Embolization of an Unruptured Aneurysm
}

BACKGROUND AND PURPOSE: Development of a headache after aneurysm coil embolization is not uncommon but has received little attention. The authors prospectively analyze the characteristics and risk factors of a headache after coiling in patients treated for an unruptured cerebral aneurysm.

MATERIALS AND METHODS: Ninety patients treated for an unruptured cerebral aneurysm over a period of 1 year, and without a headache history within a month before coiling, were enrolled in this study. All coilings were successfully performed without neurologic complications. After coiling, headache development and intensities were recorded.

RESULTS: Fifty (55.6\%) patients experienced a headache (VAS score, $4.5 \pm 2.02$ ) at 7.9 (range, 0-72) hours, on average, after coiling, and all headaches resolved within an average of 73.0 (range, 3-312) hours. Univariate analysis showed that the following were significantly associated with the development of a headache: age $\leq 50$ years (OR $4.636,95 \% \mathrm{Cl}, 1.414-15.198$ ), hypertension (OR 0.232, 95\% $\mathrm{Cl}, 0.095-0.571$ ), a packing attenuation of $>25 \%$ (OR 3.619, 95\% Cl, 1.428-9.174), and a previous headache history (OR 2.769, 95\% Cl, 1.120-6.849). However, binary logistic regression showed that only a packing attenuation of $>25 \%(P=.013$, adjusted $\mathrm{OR} 3.774,95 \% \mathrm{Cl}, 1.320-10.790)$ and no history of hypertension ( $P=.019$, adjusted $\mathrm{OR} 3.515,95 \% \mathrm{Cl}, 1.233-10.021$ ) were independently associated with the development of a headache.

CONCLUSIONS: A headache frequently developed after the coiling of unruptured aneurysms. However, headaches were relatively benign and resolved within several days. The present study shows that no hypertension history and a packing attenuation of $>25 \%$ are risk factors of headache development.

ABBREVIATIONS: $\mathrm{Cl}=$ confidence interval; NSAID = nonsteroidal anti-inflammatory drug; OR = odds ratio; $V A S=$ Visual Analogue Scale

$\mathbf{T}$ he numbers of patients treated for an unruptured aneurysm by coil embolization continue to increase, and we have observed that many patients complain of a headache after coiling. Practically, headaches that develop after coiling are benign and resolve spontaneously in most patients. In our experience, the development of a headache is not uncommon but has received little attention. We considered that patients should be given instructions before the procedure regarding headache development after coiling. The aims of this prospective study were to document the characteristics of headaches that develop after coil embolization of an unruptured aneurysm and to identify risk factors.

Received October 14, 2011; accepted after revision December 17.

From the Department of Neurosurgery (G.H., E.-A.J., J.S.B., C.W.O., O.-K.K.), Seoul National University Bundang Hospital, Seongnam, Korea; Department of Neurology (J.H.S.), Chuncheon Sacred Heart Hospital, Hallym University, Chuncheon, Korea; Department of Neurosurgery (H.P.), Jeju National University Hospital, Jeju, Korea; Department of Neurosurgery (S.-C.J.), Haeundae Paik Hospital, Inje University, Busan, Korea; and Department of Neurosurgery (B.C.K.), Dongguk University Ilsan Hospital, Goyang, Korea.

Gyojun Hwang and Eun-A Jeong equally contributed to this article as co-first authors.

This study was supported by a grant of the Korea Healthcare Technology R\&D Project, Ministry of Health \& Welfare, Republic of Korea (A102065).

Please address correspondence to 0-Ki Kwon, MD, PhD, Department of Neurosurgery, Seoul National University Bundang Hospital, 300 Gumi-dong, Bungdang-gu, Seongnam, Gyeonggi, 463-707, Korea; e-mail: meurokwonoki@gmail.com

Indicates open access to non-subscribers at www.ajnr.org

Indicates article with supplemental on-line table.

http://dx.doi.org/10.3174/ajnr.A3018

\section{Materials and Methods}

\section{Patient Collection}

After obtaining institutional review board approval for this prospective study, patients with an unruptured aneurysm who were candidates for elective endosaccular coil embolization were considered for inclusion in this study. The exclusion criteria applied were as follows: 1) a nonsaccular aneurysm, such as fusiform or dissecting aneurysm; 2) therapeutic parent artery occlusion; 3) recoiling for a recanalized aneurysm; 4) a history of subarachnoid hemorrhage; 5) a history of another cerebrovascular disease, including arteriovenous malformation and Moyamoya disease; and 6) experience of a headache during the month before coiling. Having applied these criteria, 90 consecutive patients treated from September 2009 to November 2010 were enrolled in this study.

\section{Coil Embolization Procedure}

In all patients, aneurysm coiling was performed under general anesthesia using a biplane angiographic unit (Integris Allura; Philips Healthcare, Best, the Netherlands). One neurointerventionist (O.K.K.) performed all coilings at 1 institute (Seoul National University Bundang Hospital). Preparation for, and the technical details of, the procedure were conventional and were previously described in detail. ${ }^{1-4}$ All aneurysm embolizations were performed using detachable platinum coils, including the Guglielmi (Boston Scientific, Fremont, California), MicroPlex (MicroVention, Aliso Viejo, California), and Axium (ev3, Irvine, California) coils. Modified coils, such as the Matrix (Boston Scientific) and HydroCoil (MicroVention), were not 
used. Final postembolization angiography was performed to detect thrombus formation, parent artery compromise, or distal embolism. According to the study protocol, patients with any neurologic deficit after coiling or a procedural complication, such as hemorrhage, infarction, or any other complication capable of inducing a headache, would have been excluded, but all 90 coilings were performed successfully and none of these complications were encountered.

\section{Patient Information, Aneurysmal Factors, and Procedural Factors}

Much information was collected on factors that could potentially influence headache development, such as demographic data, social background (employment status, marriage, level of education), coexistent medical conditions (hypertension, diabetes mellitus, or others), previous headaches, and medication history. In addition, information on aneurysmal characteristics (diameter, neck size, and location) and procedural factors (methods used, packing attenuation, duration of anesthesia, total radiation dose, and radiation flow time) were collected. Aneurysm diameters were classified as small ( $\leq 5 \mathrm{~mm})$, medium (5-15 mm), or large ( $\geq 15 \mathrm{~mm})$, and neck sizes were classified as narrow $(\leq 4 \mathrm{~mm})$ or wide $(>4 \mathrm{~mm})$. The following formulas were used to calculate packing attenuation:

Aneurysmal volume $(\mathrm{cc})=4 / 3 \times \pi \times[$ length $] / 2 / 10$

$$
\times[\text { width }] / 2 / 10 \times[\text { height }] / 2 / 10
$$

Coil volume $(\mathrm{cc})=\sum \pi \times([\text { coil outer diameter }] / 2 \times 2.54)^{2}$

$$
\times[\text { coil length }]
$$

Packing attenuation $(\%)=[$ coil volume $] /[$ aneurysm volume $]$

\section{Headache Details}

Headache intensities were measured using a $10-\mathrm{cm}$ horizontal VAS before and after coiling. A headache after coiling was defined as 1 or more headaches of more than mild intensity, as determined by the International Classification of Headache Disorders (2nd edition). ${ }^{5} \mathrm{~Pa}-$ tients were monitored for headache development immediately after coiling, hourly for the first 6 hours, and then every 6 hours until discharge. When a patient began to feel a headache developing, onset time, intensity, characteristics, and duration of the headache were recorded, and when a patient requested analgesics, NSAIDs (usually $650 \mathrm{mg}$ oral acetaminophen or $100 \mathrm{mg}$ aceclofenac) were administrated until the headache improved, according to European Federation of Neurological Societies guidelines. ${ }^{6}$ At 2 weeks after discharge, patients were re-evaluated for the presence of a headache at our outpatient clinic. For a patient whose headache did not resolve until discharge, we also recorded when the headache disappeared, if the headache resolved after discharge.

\section{Statistical Analyses}

Statistical analysis was conducted using SPSS version 17 (SPSS, Chicago, Illinois). The Mann-Whitney $U$ test or Wilcoxon signed rank test was used to analyze numeric variables, and the $\chi 2$ or Fisher exact test was used to analyze nominal variables. Binary logistic regression analysis was performed on variables with an unadjusted effect, with a $P$ value of $<.15$ by univariate analysis, to identify risk factors of a headache after coiling. Statistical significance was accepted for $P$ values $<.05$.

\begin{tabular}{lccc}
\hline $\begin{array}{l}\text { Risk factors of headache development after coiling of an } \\
\text { unruptured aneurysm by binary logistic regression analysis }\end{array}$ \\
\hline & Adjusted $0 \mathrm{R}$ & \multicolumn{1}{c}{$95 \% \mathrm{Cl}$} & $P$ value \\
\hline Age $\leq 50$ & 1.908 & $0.494-7.368$ & 0.349 \\
No history of hypertension & 3.515 & $1.233-10.021$ & 0.019 \\
Previous headache & 2.421 & $0.878-6.673$ & 0.087 \\
Packing attenuation $>25 \%$ & 3.774 & $1.320-10.790$ & 0.013 \\
\hline
\end{tabular}

\section{Results}

Of the 90 patients enrolled in this study, 50 (55.6\%) experienced a headache after coiling (mean maximum VAS score, $4.5 \pm 2.02$ ). Headaches developed, on average, at 7.9 hours (range, 0-72) after coiling, and all resolved within an average of 73.0 hours (range, 3-312). The characteristics of these headaches were a dull generalized pain $(n=20,40.0 \%)$, a throbbing pain $(n=15,30.0 \%)$, tightness $(n=7,14.0 \%)$, a shooting pain $(n=4,8.0 \%)$, and others $(n=4,8.0 \%)$. NSAIDs were prescribed in $40(80 \%)$ of the 50 patients who developed a headache. The total amount of NSAIDs was as follows: no medication in 11 (22\%); acetaminophen $<3.25 \mathrm{~g}$ or aceclofenac $<0.5 \mathrm{~g}$ in $21(42 \%)$; aceclofenac $0.5-1 \mathrm{~g}$ in 9 (18\%); aceclofenac $\geq 1 \mathrm{~g}$ in 9 (18\%). The mean VAS scores of patients treated with NSAIDs before and 24 hours after prescription were $4.5 \pm 1.90$ and $3.3 \pm 2.25$, respectively. NSAIDs significantly lowered VAS scores within 24 hours $(P<.001)$, but headaches persisted in $33(82.5 \%)$ patients. NSAIDs were prescribed for a mean of 2.5 days (range, $0.5-12.5$ ), that is, $\leq 12$ hours in $16(40 \%), 12-24$ hours in $3(7.5 \%), 1-3$ days in $9(22.5 \%), 3-7$ days in $9(22.5 \%)$, and $>7$ days in $3(7.5 \%)$ patients.

Basic clinical data, social backgrounds, aneurysmal characteristics, and procedure-related factors are summarized in the On-line Table. Patients who developed a headache (the headache group) were younger $(54.7 \pm 12.36$ versus $60.5 \pm 8.43$ years; $P=.011)$ and more likely to be $\leq 50$ years old $(34.0 \%$ versus $10.0 \%, P=.011$; OR 4.636, 95\% CI, 1.414-15.198). In terms of coexistent medical conditions, the proportion of patients with hypertension was smaller in the headache group than in the nonheadache group $(38.0 \%$ versus $72.5 \%, P=$ .001 ; OR 0.232, 95\% CI, 0.095-0.571). Furthermore, the proportion of patients with a previous headache history (usually a migraine type headache) was significantly higher in the headache group (48.0\% versus $25.0 \%, P=.030$; OR $2.769,95 \% \mathrm{CI}$, $1.120-6.849)$. Interestingly, packing attenuation was higher in the headache group $(30.0 \pm 7.01 \%$ versus $25.7 \pm 7.84 \%$, $P=.007)$, and aneurysms with a packing attenuation of $>25 \%$ were significantly more frequent in the headache group (80.0\% versus $52.5 \%, P=.007$; OR 3.619, 95\% CI, $1.428-$ 9.174). Social backgrounds, aneurysmal characteristics, method used, anesthesia time, radiation dose, and radiation flow time were not found to be significantly different in these 2 groups.

Binary logistic regression analysis showed that a packing attenuation of $>25 \%(P=.013$, adjusted OR $3.774,95 \% \mathrm{CI}$, $1.320-10.790)$ and no history of hypertension $(P=.019$, adjusted OR 3.515, 95\% CI, 1.233-10.021) were significantly associated with the development of a headache after coiling (Table). 


\section{Discussion}

This series shows that a headache developed in approximately half of our patients after coil embolization of an unruptured aneurysm. However, all headaches were benign and resolved on average at 3 days after coiling. The absence of a history of hypertension and a packing attenuation of $>25 \%$ were found to be associated with the occurrence of a headache after coiling. In our practice, these findings are being used to provide information to patients before and after coiling.

Dilation or stretching of intracranial arteries can produce a headache. ${ }^{7-16}$ This dilation can be induced by mechanical forces as well as chemicals. It is well known that many patients experience a headache during angioplasty of the intracranial artery, ${ }^{7,8,13}$ and a similar mechanism may apply to headache development after coil embolization. During and after coiling, if coil packing is enough to entrap blood and to not permit free blood flow, thrombosis can be induced in the coil frame and aneurysmal sac. The coil mass itself, and thrombosis in coiled aneurysms, cause distension of the aneurysmal wall, which can induce a headache. In addition, this thrombosis was also found to lead to local inflammation and subsequent headache. ${ }^{17}$ These appear to explain the finding that headaches develop more frequently with a high $(>25 \%)$ packing attenuation.

Healthy vessels unaffected by hypertension could be more distensible and elastic, ${ }^{18-21}$ and thus the walls of aneurysms in patients without a hypertension history could be more affected by pressure increases within aneurysmal sacs, and may explain why patients without a hypertension history had a greater rate of headache development.

The present study has several limitations that warrant consideration. First, the study is limited by its relatively small cohort, which may have affected our analysis of some of the factors investigated. For example, some aneurysm locations were not well represented, especially aneurysms in posterior circulation, and thus we were not able to analyze the effect of location. Second, VAS may not be the best measure of headache intensity. However, VAS has most of characteristics that an ideal method for pain measurement should have. ${ }^{22}$ In addition, in our experience before conducting this study, a headache after coiling was usually mild or moderate. VAS is known to be suitable for trials that include patients with mild and moderate headaches. ${ }^{23,24}$ Furthermore, few patients who are candidates for elective coil embolization have factors impeding scale use, such as impaired abstract thinking ability or psychomotor problems. ${ }^{25-27}$ Therefore, we chose VAS as a measurement tool of headache intensity. Finally, MR imaging was not performed after coiling to detect any other reasons that can cause a headache, including small embolic infarctions. However, a review of the literature failed to unearth any relationship between small infarctions and headaches. If MR imaging were performed in all of our study subjects, it would be more helpful to prove the relationship between thrombosis and inflammation, and the development of a headache after coiling. However, because the study reporting this finding was recently published, ${ }^{17}$ we were unable to consider MR imaging evaluation for this purpose. Nevertheless, our findings demonstrate that headaches after coiling are benign, short-lived, and easily controlled. We hope that these results will be used by physicians to provide information about headache devel- opment to patients before and after coiling of an unruptured aneurysm.

\section{Conclusions}

A headache develops in approximately $50 \%$ of patients with an unruptured aneurysm after coiling. However, headaches are usually benign, short-lived, and easily controlled. This study shows that absence of a hypertension history indicates susceptibility to the development of a headache, and that a high coil packing attenuation is also a risk factor of headache development after coiling.

\section{References}

1. Hwang G, Jung C, Park SQ, et al. Thromboembolic complications of elective coil embolization of unruptured aneurysms: the effect of oral antiplatelet preparation on periprocedural thromboembolic complication. Neurosurgery 2010;67:743-48; discussion 748

2. Hwang G, Jung C, Sheen SH, et al. Two-year follow-up of contrast stasis within the sac in unruptured aneurysm coil embolization: progressive thrombosis or enlargement? AJNR Am J Neuroradiol 2010;31:1929-34

3. Im SH, Han MH, Kwon OK, et al. Endovascular coil embolization of 435 small asymptomatic unruptured intracranial aneurysms: procedural morbidity and patient outcome. AJNR Am J Neuroradiol 2009;30:79-84

4. Kwon OK, Kim SH, Kwon BJ, et al. Endovascular treatment of wide-necked aneurysms by using two microcatheters: techniques and outcomes in 25 patients. AJNR Am J Neuroradiol 2005;26:894-900

5. Headache Classification Subcommitee of the International Headache Society. The International Classification of Headache Disorders (2nd ed.). Cephalalgia 2004;24:9-160

6. Evers S, Afra J, Frese A, et al. EFNS guideline on the drug treatment of migraine-report of an EFNS task force. Eur Journal Neurol 2006;13:560-72

7. Abou-Chebl A, Krieger DW, Bajzer CT, et al. Intracranial angioplasty and stenting in the awake patient. J Neuroimaging 2006;16:216-23

8. Beekman R, Nijssen PC, van Rooij WJ, et al. Migraine with aura after intracranial endovascular procedures. Headache 2001;41:410-13

9. Gil-Gouveia RS, Sousa RF, Lopes L, et al. Post-angiography headaches. J Headache Pain 2008;9:327-30

10. Graham JR, Wolff HG. Mechanism of migraine headache and action of ergotamine tartrate. Arch Neuro Psychiatry 1938;39:737-63

11. Iversen $\mathrm{HK}$, Nielsen $\mathrm{TH}$, Olesen J, et al. Arterial responses during migraine headache. Lancet 1990;336:837-39

12. Lauritzen M, Olesen J. Regional cerebral blood flow during migraine attacks by Xenon-133 inhalation and emission tomography. Brain 1984;107:447-61

13. Martins IP, Baeta E, Paiva T, et al. Headaches during intracranial endovascular procedures: a possible model of vascular headache. Headache 1993;33:227-33

14. May A, Goadsby PJ. The trigeminovascular system in humans: pathophysiologic implications for primary headache syndromes of the neural influences on the cerebral circulation. J Cereb Blood Flow Metab 1999;19:115-27

15. Olesen J, Larsen B, Lauritzen M. Focal hyperemia followed by spreading oligemia and impaired activation of rCBF in classic migraine. Ann Neurol 1981;9:344-52

16. Thomsen LL, Iversen HK, Olesen J. Cerebral blood flow velocities are reduced during attacks of unilateral migraine without aura. Cephalalgia 1995;15:109-16

17. Berge J, Tourdias T, Moreau JF, et al. Perianeurysmal brain inflammation after flow-diversion treatment. AJNR Am J Neuroradiol 2011;32:1930-34

18. Boulanger CM. Secondary endothelial dysfunction: hypertension and heart failure. J Mol Cell Cardiol 1999;31:39-49

19. De Silva DA, Woon FP, Gan HY, et al. Arterial stiffness is associated with intracranial large artery disease among ethnic Chinese and South Asian ischemic stroke patients. J Hypertens 2009;27:1453-58

20. Kotsis V, Stabouli S. Arterial stiffness, vascular aging, and intracranial large artery disease. Am J Hypertens 2011;24:252

21. Ryan SM, Waack BJ, Weno BL, et al. Increases in pulse pressure impair acetylcholine-induced vascular relaxation. Am J Physiol 1995;268:H359-63

22. Price DD RJ, Wade JB. Psychophysical approaches to measurement of the dimensions and stages of pain. London: Guilford Press;2001:53-75

23. Bendtsen L, Bigal ME, Cerbo R, et al. Guidelines for controlled trials of drugs in tension-type headache: second edition. Cephalalgia 2010;30:1-16

24. Tfelt-Hansen P, Block G, Dahlof C, et al. Guidelines for controlled trials of drugs in migraine: second edition. Cephalalgia 2000;20:765-86

25. Herr KA, Mobily PR. Complexities of pain assessment in the elderly. Clinical considerations. J Gerontol Nurs 1991;17:12-19

26. Jensen MP, Karoly P, Braver S. The measurement of clinical pain intensity: a comparison of six methods. Pain 1986;27:117-26

27. Kremer E, Atkinson JH, Ignelzi RJ. Measurement of pain: patient preference does not confound pain measurement. Pain 1981;10:241-48 